\begin{tabular}{|c|c|}
\hline \multirow{3}{*}{ 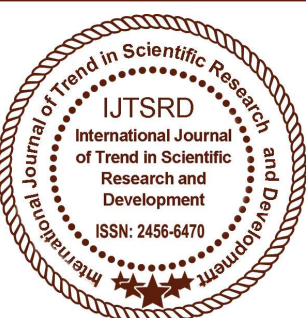 } & $\begin{array}{l}\text { International Journal of Trend in Scientific } \\
\text { Research and Development (IJTSRD) }\end{array}$ \\
\hline & Access Journal \\
\hline & ISSN No: 2456 - 6470 | www.ijtsrd.com | Volume - 2 | Issue -3 \\
\hline
\end{tabular}

\title{
An Effective Routing Algorithm to Enhance Efficiency with WSN
}

\author{
Anita Gracios \\ M.Tech Scholar, CSE Department, SIRT Bhopal, \\ Madhya Pradesh, India
}

\author{
Prof. Arun Jhapate \\ Asst. Prof., Department of CSE, SIRT, Bhopal \\ Madhya Pradesh, India
}

\begin{abstract}
Moving network is the most valuable network of today environment since it does multiple and multilevel communication services during move, it also incorporate many digital techniques to performance real time confidential and security awareness task for the purpose of user facility and services in the similar way it generate various challenges for its designer and developer to make then trustworthy and reliable, it is also one of the major technique to improve the user application based days to days business services and its control, to make them more effective and trustworthy we need to develop secure routing and performance in the WSN system so that everyone can trust on the mobile devices to go for the multiple transaction as required time to time.
\end{abstract}

We have developed a new efficient routing model that not only improve routing in WSN but also better in performance level compare to the traditional protocols like AODV and DSR routing algorithm we had implement the proposed routing algorithm at NS2 and wonderfully find the most awaited better result the not the other previously proposed techniques, therefore one can say it is the trust based computing algorithm for WSN to enhance the working capacity of WSN and reliability parameters. The specially of this model it is support dynamic moving network for boosting the performance among the nodes as well as route request with maximum bandwidth to reach towards its required destination, another good aspect of this is to give better security environment along with safeguard to overcome the previous challenges and issues occurred in traditional implementation and algorithm.
Keywords: $A O D V, D S R$, Routing, NS2, WSN

\section{INTRODUCTION}

The proposed Routing model is very important for future aspect as well as present since it has been design with the possible challenges of near future and the WSN is growing through multiple application and movable devices so that it can be possible to adopt high number of request with minimum overhead and to take the responsibility of handing the traffic pattern as well as the networking scenario that counterpart the problem regarding different category of network and traffic pattern also it need to be understand the factors of new communication era, our model will take care the responsibility to handle large number of signals along with delay factor and performance management parameters all of the thing has been taken care so that process of management of signals through WSN can be achieved in this way a new era of networking has been developed and further aspect of communication can be managed, other parameters of such type of reactive routing technique just to provides and easiest way of user communication interface that enable a function to adopt new things and also perform the best result over the performance factors, in this coming session we will explain the implementation part of AODV,DSR and new algorithm who will compare the performance factors with the variations in numbers of the node we perform the concept of AODV ,DSR and proposed model similar way at the end we find there is a drastically changes has been highlighted through different graph lines as in [1]author defines that in WSN different node will communicate to the other nodes for information sharing, many times one found that node or network failure has been caught at 
multiple location of moving network in this way we can say the proposed implementation module has been capable enough to handle the things and only the way to provides mart solution for complex traffic pattern for taking such a beautiful communication system and the information flow for the network point of view, this causes high an d low for the demanding system security and management skills.

\section{RELATED WORK}

Traditionally various reactive routing techniques has been implemented but it is also true that no protocols or algorithm is yet applicable to avoid data loss and high performance issues in which few of them .

\section{A. DSR Routing}

It is one of the most common routing protocols of wireless networking that has been implemented, protocols focus for small wireless network to get obtained the high throughput for low number of network with only a single dedicated server node will serve the communication services among the all other in order to provide efficient secure services in which we observed that DSR is well enough to manage small wireless network with limited number of nodes since it support multi-hop communication system in wireless network since DSR is comes in Reactive routing therefore it is also necessary to perform routing on demand basis with surprise wireless networking situation.

\section{B. Routing in DSR}

It is very necessary to know the procedure of performing initial routing at the beginning at the process to get initialize the network with multiple numbers of networks.DSR will perform routing in a manner in which packets has been requested first from actual sender and receiver system then only, in this case special packet has been generated from sender node called RREQ which will be broad casted in further steps for successful completion, in receiver node have original destination address will find the request and response against this, the same process will continue until the TTL is greater than others.

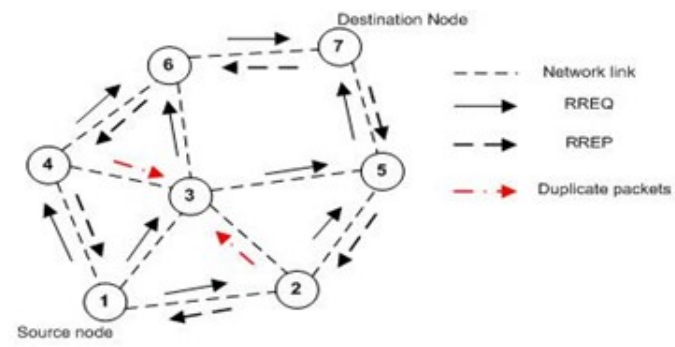

Figure 1.1: Routing In DSR

\section{Route Maintenance DSR}

DSR is good for link recovery when ever any connection gets losses, as the figure 1.2 implies that DSR process the necessary process in order to perform routing with securely and efficiently, with low overheads and maintenance along the normal number of nodes.

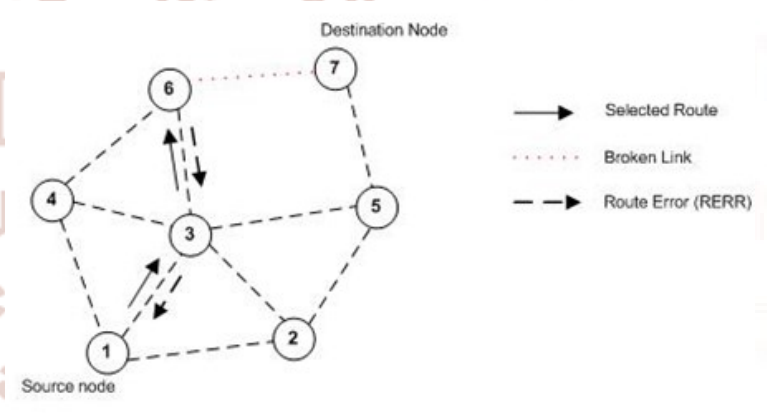

Figure 1.2: Route Maintainnace in DSR

\section{Implementation of DSR at NS2}

Figure 1.3 (a) and (b) DSR implementation defines there is only one node (0) has itseft works as server going through the destination node during this 0 node will interact to their neighbor nodes for the services, DSR perform well with limited no. of nodes as shown in figure $1.3(\mathrm{~b})$.

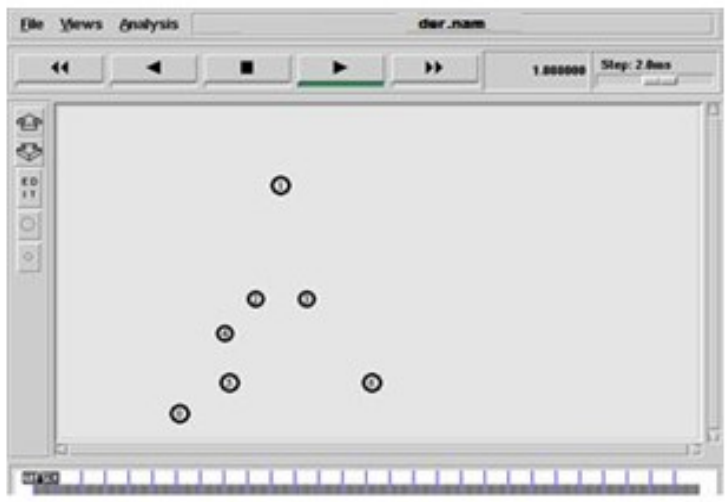

(A) 


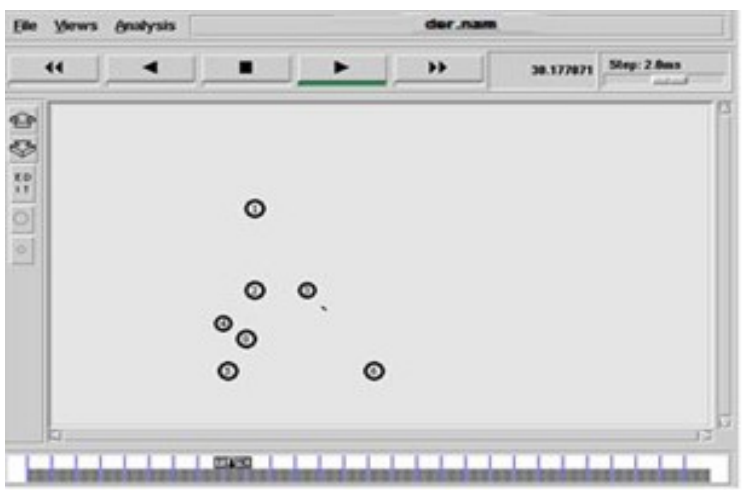

(B)

Figure 1.3: DSR nodes behavior at NS2

In figure 1.4 (a) and (b) we observed that DSR is good for limited no. of nodes in wireless networking we observe node 0 behave like server to find route and perform data transmission services but it is also observe after some time networking suffering through data loss issues.
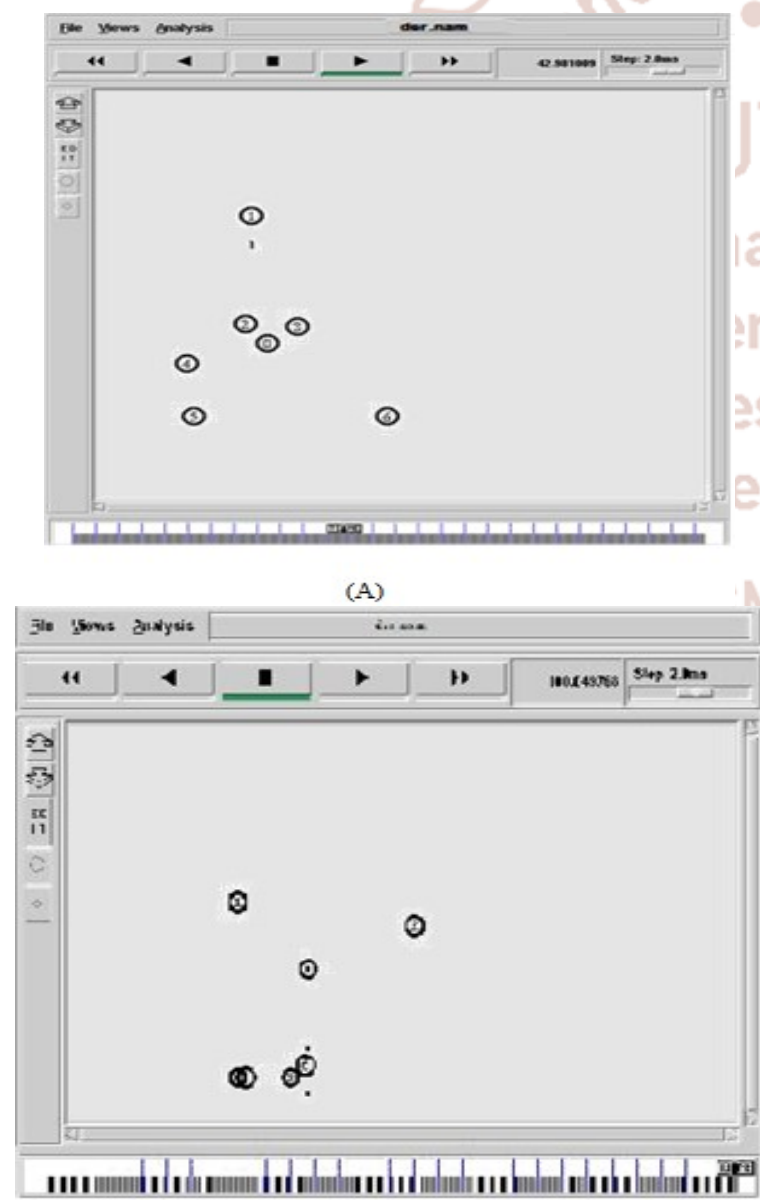

(B)

Figure 1.4: Observing Data Loss at NS2

In this way we observer the performance of DSR with dynamic wireless network at NS2, DSR suffer with data loss issues at some point. In this way we need more reliable algorithm so that we can save bandwidth and perform efficient routing and management with low or high number of nodes.

\section{IMPLEMENTATION OF ROUTING MODEL AT NS2 \\ PROPOSED}

In figure $1.5(\mathrm{~A})$ and (B) authors experimenting the implementation detail of proposed model with server 1 and server 2 . In this process server 1 is dedicatedly available at the node zone to perform routing over their networking area and server 2 will take care the responsibility of handling the reaming node who participating through outside network zone area.
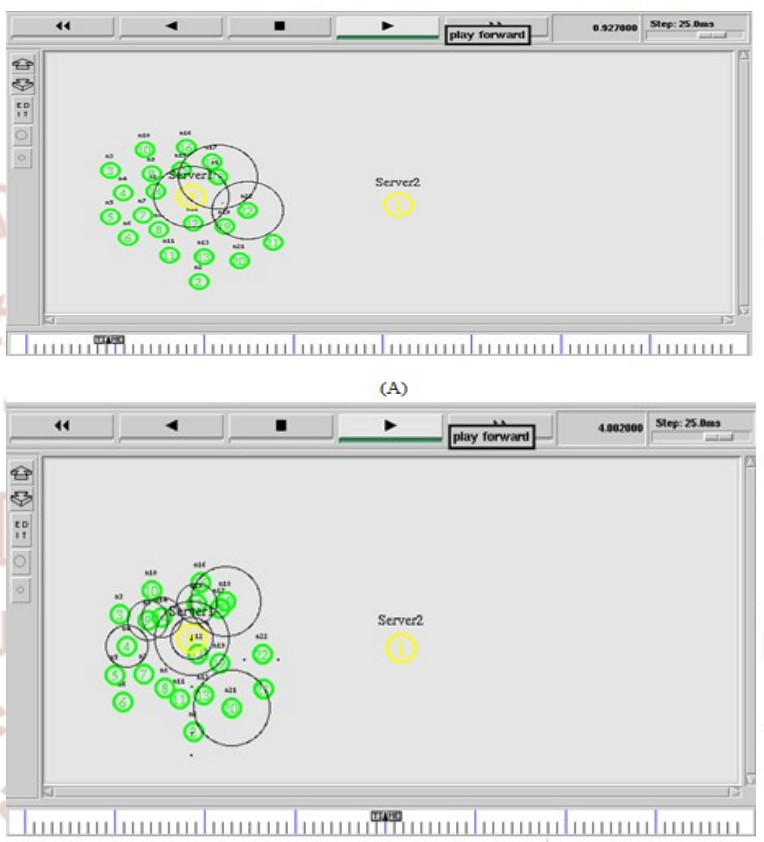

(B)

Figure 1.5: Simulating Proposed Routing Model at NS2

In figure 1.6 (a) and (b) define that proposed algorithm is working find with the responsibility of handling multiple node at the same time and also manage the traffic load in distributed way.

In figure 1.6 (a) server 1 taking the role of wireless distributed services as soon as the server 1 moves over the assign zone, the frequently occurred moving nodes has been introduced which has been managed by server 2 showing in figure 1.6 (b).

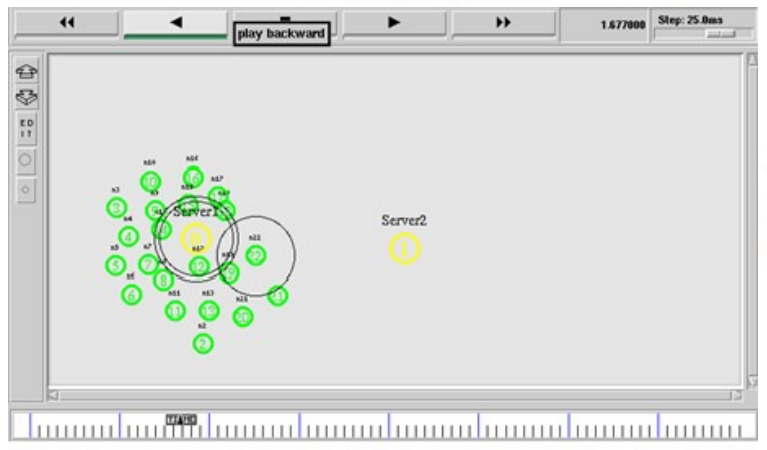

(A) 
International Journal of Trend in Scientific Research and Development (IJTSRD) ISSN: 2456-6470

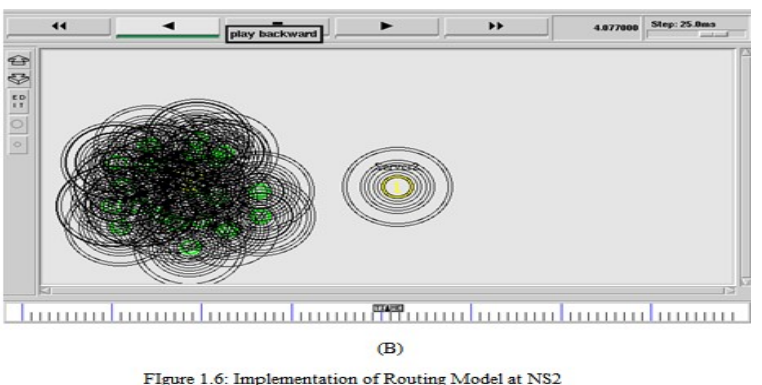

\section{CONCLUSION}

In previous section we going through deep study of AODV ,ADS and Proposed Routing Model at NS2 at implementation level, we perform the wireless network with $1000 \times 1000$ area along with variations in number of nodes we find that AODV having performance losses during the process, in DSR we find data has been lost due to heavy load and complex networking scenario and in Proposed model we found a superior way to get perform efficient routing and traffic distribution through server 1 and server 2 , which behave similar to each and every node for efficient routing and protecting data loss when load gets higher.

\section{REFERENCES}

1) F. Stajano and R. J. Anderson. "The resurrecting duckling: Security issues for ad-hoc wireless networks" In 7th Security Protocols Workshop, volume 1796 of Lecture Notes in Computer Science, Cambridge, United Kingdom, 1999. Springer-Verlag, Berlin Germany.

2) Dirk Balfanz, D. K. Smetters, Paul Stewart and H. Chi Wong: "Talking To Strangers: Authentication in Ad-Hoc Wireless Networks", Symposium on Network and Distributed Systems Security (NDSS'02), Xerox Palo Alto Research Center, Palo Alto, USA, 2002.

3) DINA S. DEIF “An Ant Colony Optimization Approach for the Deployment of Reliable Wireless Sensor Networks" Received May 15, 2017, accepted May 29, 2017, date of publication June 5, 2017, date of current version June 27, 2017.Digital Object Identifier 10.1109/ACCESS.2017.2711484.

4) DANYANG QIN" Research on Trust Sensing Based Secure Routing Mechanism for Wireless Sensor Network" Received April 17, 2017, accepted May 16, 2017, date of publication May 23, 2017, date of current version June 28, 2017.
Digital

Object

Identifier

10.1109/ACCESS.2017.2706973.

5) YA GAO, "Heterogeneous Statistical QoS Provisioning Over Wireless Powered Sensor Networks" Received March 14, 2017, accepted March 31, 2017, date of publication April 13, 2017, date of current version June 7, 2017. Digital Object

Identifier 10.1109/ACCESS.2017.2694046.

6) Quality of Service of Routing Protocols in Wireless Sensor Networks: A Review" Received December 20, 2016, accepted January 7, 2017, date of publication January 17, 2017, date of current version March 13, 2017. Digital Object Identifier 10.1109/ACCESS.2017.2654356.

7) Wang $\mathrm{Na}$ "Trust Model Based on Changeable Sampling Frequency for Wireless Sensor Network" 2016 IEEE ICIS 2016, June 26-29, 2016, Okayama, Japan.

8) Quan Wang"Comparative Examination on Architecture and Protocol of Industrial Wireless Sensor Network Standards" IEEE COMMUNICATIONS SURVEYS \& TUTORIALS, VOL. 18, NO. 3, THIRD QUARTER 2016.

9) KATSUYA SUTO "An Energy-Efficient and Delay-Aware Wireless Computing System for Industrial Wireless Sensor Networks" Received April 3, 2015, accepted April 21, 2015, date of publication June 15, 2015, date of current version July 17, 2015. Digital Object Identifier 10.1109/ACCESS.2015.2443171.

10) Md Nafees Rahman "Efficient Algorithm For Prolonging Network Lifetime Of Wireless Sensor Networks" Tsinghua Science And Technology Issnll1007-02141101/12llpp561-568 Volume 16, Number 6, December 2011.

11) J. Kong, P. Zerfos, H. Luo, S. Lu, and L. Zhang. "Providing robust and Ubiquitous Security support for Mobile Ad Hoc Networks ", Proceedings of the 9th International conference on Network Protocols (ICNP), Riverside, California, USA, November 11-14 2001.

12) Edith C. H. Ngai and Michael R. Lyu. "Trust- and Clustering-Based Authentication Services in Mobile Ad Hoc Networks", 24th International Conference on Distributed Computing Systems Workshops - W4: MDC (ICDCSW'04), Hachioji, Tokyo, Japan, 3/23-24, 2004. 